Document downloaded from:

http://hdl.handle.net/10251/150461

This paper must be cited as:

Llorente, R.; Morant, M.; Puche, JF.; Romme, J.; Amiot, N.; Uguen, B.; Duplicy, J. (2010). Localization and Fingerprint of Radio Signals Employing a Multichannel Photonic Analog-toDigital Converter. IEEE Transactions on Microwave Theory and Techniques. 58(11):33043311. https://doi.org/10.1109/TMTT.2010.2076730

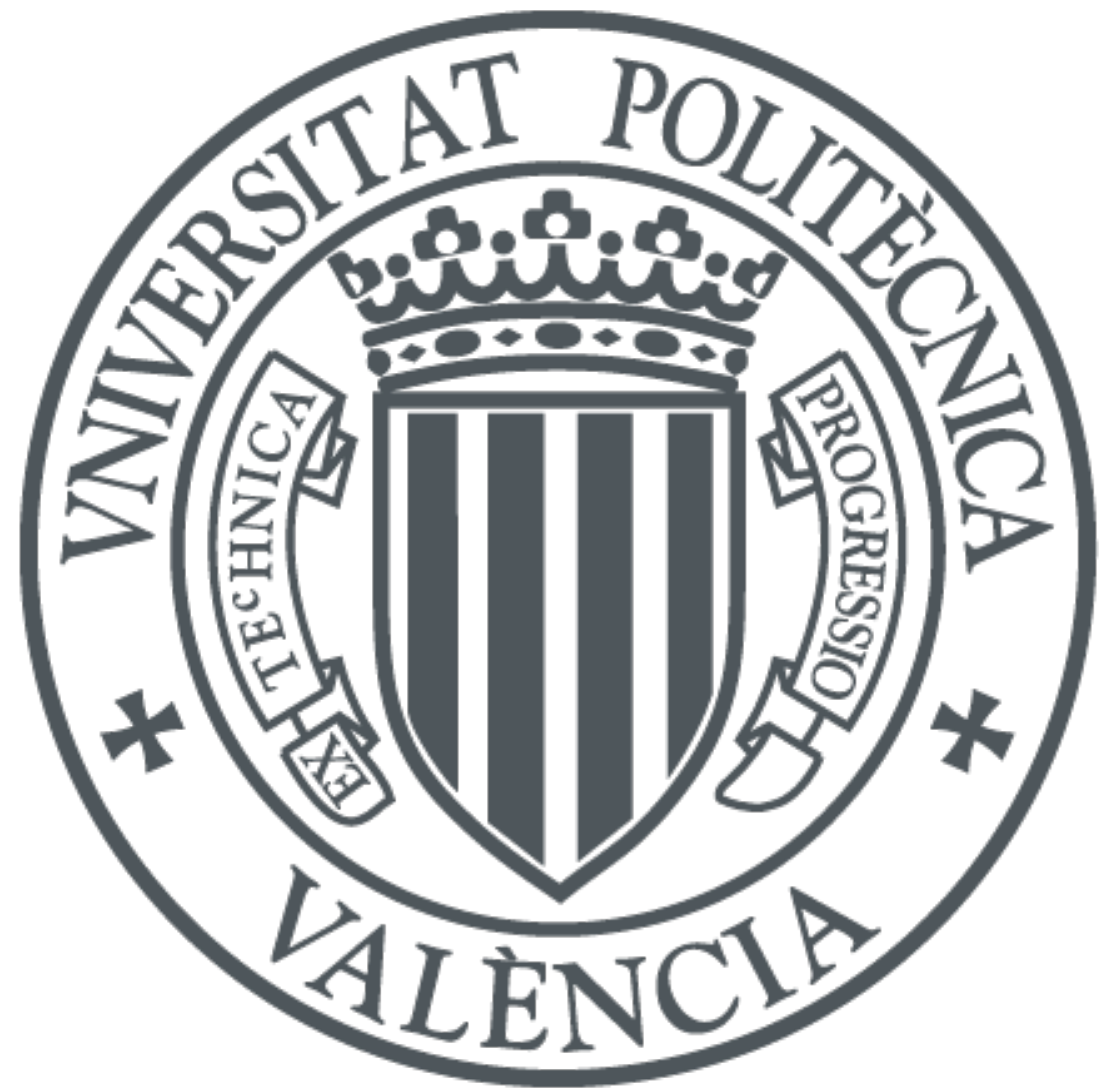

The final publication is available at

https://doi.org/10.1109/TMTT.2010.2076730

Copyright Institute of Electrical and Electronics Engineers

Additional Information 


\title{
Localization and Fingerprint of Radio Signals Employing a Multichannel Photonic Analog-to-Digital Converter
}

\author{
Roberto Llorente, Member, IEEE, Maria Morant, Student Member, IEEE, José F. Puche, Jac Romme, \\ Nicolas Amiot, Bernard Uguen, Member, IEEE, and Jonathan Duplicy, Member, IEEE
}

\begin{abstract}
The fingerprint and localization of radio signals employing a multichannel photonic analog-to-digital converter (ADC) is proposed, analyzed and demonstrated in a laboratory experiment. The photonic-ADC detects the radio signals with high sensitivity in a large bandwidth without down-conversion stages. This is of special interest when processing emerging lowpower wireless standards like ultra-wideband radio. The optical processing in the multichannel photonic-ADC is tailored for the localization and fingerprint of generic radio transmitters when orthogonal-frequency division multiplexing (OFDM) modulation is employed in the transmission. The photonic-ADC includes engineered optical and electrical amplification. The experimental work demonstrates that detection of radio signals with $-65 \mathrm{dBm}$ power with signal-to-noise ratio better than $20 \mathrm{~dB}$ is feasible, being in good accordance with the theoretical analysis. The multichannel photonic-ADC comprises five optical channels which are precisely time-aligned in optical domain achieving $0.23 \mathrm{~m}$ spatial resolution (median) in the localization of radio transmitters. The experimental work also demonstrates that photonic-ADC processing is adequate for OFDM-based ultrawideband radio signal fingerprint including estimation of the average power, frequency band of operation and time-frequency hopping pattern if applicable. UWB transmitter localization has been experimentally demonstrated with $0.4 \mathrm{~m}$ error.
\end{abstract}

Index Terms- Multichannel photonic analog to-digital converter, ultra-wideband radio sensing, orthogonal-frequency division multiplexing, fingerprinting, wireless transmitter localization

Manuscript received January 1, 2010, revised July 9, 2010. This work was supported in part by the European $7^{\text {th }}$ Framework Program project UCELLS FP7-IST-216785. M. Morant's work is supported by Spain FPU MEC grant AP2007-01413.

R. Llorente and M. Morant are with the Nanophotonics Technology Centre of the Universidad Politécnica de Valencia, Camino de Vera s/n, 46022 Valencia, Spain (e-mail: rllorent@ntc.upv.es; mmorant@ntc.upv.es).

J. F. Puche is with DAS Photonics S.L., Camino de Vera s/n, 46022 Valencia, Spain (email: jpuche@ dasphotonics.com).

J. Romme is with IMST GmbH, Carl-Friedrich-Gauss-Str. 2, 47475 KampLintfort NRW, Germany (e-mail: romme@imst.de).

N. Amiot and B. Uguen are with the Institut d'Electronique et de Télécommunications de Rennes, UMR CNRS 6164, Rennes, France (e-mail: nicolas.amiot@univ-rennes1.fr; bernard.uguen@univ-rennes1.fr).

J. Duplicy is with Agilent Technologies Laboratories, Wingepark 51, 3110 Rotselaar, Belgium (e-mail: jonathan_duplicy@agilent.com).

\section{INTRODUCTION}

SENSING radio signals is a challenging task when the $N_{\text {signal to be detected exhibits simultaneously a very-low }}$ power level, a large bandwidth or just a broad -sometimes unknown- spectral allocation. Signal detection in such circumstances has been the typical case in electronic intelligence applications. Moreover, this application scenario is recently found in cognitive radio techniques [1] considering emerging ultra-low power low-consumption radio [2].

Detection and processing of ultra-low power radio with very large bandwidth is limited by the tradeoff between the effective analog bandwidth and equivalent number of bits (ENOB) in current electronic analog-to-digital converters (E-ADC) technology [3]. State-of-the-art E-ADCs exhibit a dynamic range too limited for monitoring ultra-low power radio in presence of other radio signals with higher radiated power levels. Optical sampling permits simultaneously high sampling rate altogether a large dynamic range [4]. Regarding sampling rate, market-available stand-alone E-ADC state-ofthe-art is Agilent-Acqiris DC252HF exhibiting 8 GSamples/s with $3 \mathrm{GHz}$ analog bandwidth. Depending on the input signal amplitude, these ADC exhibit an effective number-of-bits of approximately 5 to 6.3 bits, measured over the full bandwidth. E-ADCs state-of-the-art, regarding dynamic range (singlechannel operation), is ATMEL $2.2 \mathrm{GSamples} / \mathrm{s}$ with 7.7 ENOB. Photonics can be applied to ADC technology improving the ENOB compared to conventional E-ADCs with the same equivalent electrical bandwidth [4], or extending the bandwidth of operation maintaining the ENOB [5].

Photonic-ADC (Ph-ADC) techniques are of special interest when sensing ultra-wideband (UWB) radio signals. UWB radio is quickly entering in the market addressing wireless connection of peripherals and high-definition audio/video wireless streaming [6]. UWB radio exhibits a regulated spectrum allocation from 3.1 to $10.6 \mathrm{GHz}$ with a minimum bandwidth of $500 \mathrm{MHz}$ or $20 \%$ fractional bandwidth [7]. The maximum equivalent isotropically radiated power allowed in current regulation is $-41.3 \mathrm{dBm} / \mathrm{MHz}$, i.e. a very low power level is actually available for sensing UWB transmitters located far away. The characteristics of UWB radio imply that detection of UWB signals in the regulated band with market- 
available state-of-the-art E-ADCs is not possible in real-time, and filtering and down-conversion stages are required [8].

This letter proposes a multichannel photonic-ADC with engineered optical and electrical amplification for sensing OFDM-based UWB signals [9]. This architecture permits sampling the signal captured simultaneously from different sensors with only a single E-ADC and the time-stretching compresses the electrical spectrum and relaxes the requirements of bandwidth of the E-ADC and receiver amplifiers. The signals detected by the multichannel photonicADC are further processed to localize and fingerprint the UWB transmitters present in a given area.

The letter is organized in five sections. Section II describes the photonic-ADC technique and its performance is evaluated by simulation. In Section III the signal-to-noise ratio of the single-channel $\mathrm{Ph}-\mathrm{ADC}$ is investigated experimentally and compared with simulation results. Section IV describes the fingerprinting and localization algorithms elaborated to analyze the data sensed and processed by the Ph-ADC. A study of positioning error depending on the location of the sensors in a room is included in this section, and an experimental demonstration of the five-channel multiplexed photonic-ADC system is discussed. Finally, Section V summarizes the main conclusions from this work.

\section{TIME-STRETCH PHOTONIC-ADC PRINCIPLE AND EXPECTED PERFORMANCE}

The multichannel photonic-ADC system herein proposed is based on the optical time-stretching technique reported in [5]. The basic principle can be summarized as follows: The radio signal under analysis is modulated on a pulsed laser light which is optically time-stretched prior to digitization with a conventional E-ADC. The time-stretch process introduces three key advantages: (i) The E-ADC sampling rate, and its analog bandwidth, is effectively multiplied by a factor exactly equal to the optical stretching factor $M$. (ii) The overall system jitter is dominated by the pulsed laser jitter, typically much lower than jitter in conventional E-ADCs [4]. (iii) The E-ADC dynamic range is maintained if the $\mathrm{Ph}-\mathrm{ADC}$ is properly designed, i.e. optical distortion is limited. The time-stretching process implies that the temporal duration of the laser optical pulses is increased and a guard-time between pulses must be considered when designing the system. This implies that continuous operation would require parallel braches as described in [10]. In our case, this is not an important limitation as considerable computational efforts are required by the localization and fingerprinting algorithms, already constraining continuous operation. The time-stretching $\mathrm{Ph}$-ADC approach is especially interesting for sensing ultralow power radio as it permits the engineering of the required amplification between optical and electronic domains relaxing individual amplifier requirements.

Fig. 1 shows a single channel Ph-ADC based on the timestretching technique. This figure shows a radio signal detected by the antenna-amplifier-modulator chain, marked "radio

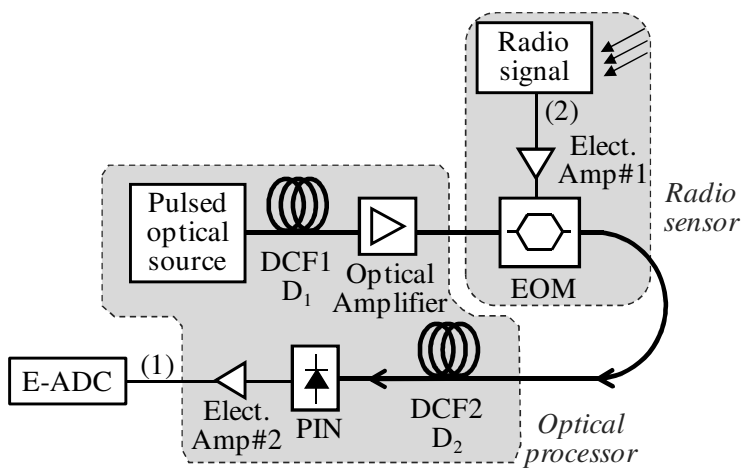

Fig. 1. Block diagram of the single channel Ph-ADC evaluated by simulation sensor" in the figure, and a photonic part implementing the time-stretch process, marked as "optical processor". The radio sensor requires an electro-optic modulator (EOM) for the radio signal conversion to optical domain, and two dispersion compensation fiber (DCF) spools with accumulated chromatic dispersion $D_{1}$ and $D_{2}$ respectively. The resulting Ph-ADC time-stretching factor is then $M=1+D_{2} / D_{l}$.

The time-stretch Ph-ADC operation can be described as follows: The accumulated chromatic dispersion of the first fiber spool $\left(D_{l}\right)$ converts the spectral components of the pulsed optical source to time domain ( $\lambda$-to-t mapping) process as described in [5]. This process is based on the temporal analogy of the Fraunhofer spatial diffraction. This approach requires the propagation of the optical pulses through a highly dispersive element (chromatic dispersion) meeting the condition $\left|T^{2} / \ddot{\Phi}\right| \ll 1$ [11], where $\ddot{\Phi}\left[\mathrm{ps}^{2} / \mathrm{rad}\right]$ stands for the chromatic dispersion in the dispersive element, and $T$ stands for $1 / \mathrm{e}$ pulse width from pulsed optical source. In our implementation, dispersion compensating fiber (OFS LLMicroDK) is employed as dispersive element. After the $\lambda$-to-t mapping, the EOM modulates the radio signal under detection. The second fiber spool, DCF2, stretches the modulated optical pulses in time domain. At the receiver end the modulated and stretched pulses are photo-detected, amplified and digitized by a conventional E-ADC.

Let us analyze the Ph-ADC expected performance. A simulation analysis employing Matlab ${ }^{\mathrm{TM}}$ targeting to evaluate the distortion added by the time-stretch process and the associated signal-to-noise ratio has been done. A radiofrequency (RF) sinusoid is considered as the radio signal under detection. The parameters characterizing the blocks in Fig. 1 in

TABLE I

SIMULATION PARAMETERS

\begin{tabular}{l|l|l|l}
\hline \hline Parameter & Value & Parameter & Value \\
\hline $\begin{array}{l}\text { DCF1 accumulated } \\
\text { dispersion (GVD) }\end{array}$ & $-1513.1 \mathrm{ps} / \mathrm{nm}$ & Elect. Amp\#1 & G=40 dB \\
DCF2 accumulated & & Gain and Noise & $\mathrm{NF}=3 \mathrm{~dB}$ \\
dispersion (GVD) & $-3612.2 \mathrm{ps} / \mathrm{nm}$ & Elect. Amp\#2 & $\mathrm{G}=62 \mathrm{~dB}$ \\
$\mathrm{~V} \pi$ modulator & $1.5 \mathrm{~V}$ & Gain and Noise & $\mathrm{NF}=2.8 \mathrm{~dB}$ \\
& PIN responsivity & $0.7 \mathrm{~A} / \mathrm{W}$ \\
\hline \hline
\end{tabular}


are summarized in Table I. Distortion is calculated comparing the simulation results with a-priori information (amplitude, frequency and phase) on the modulating RF signal at the E-ADC, point (1) in Fig. 1. The simulation sampling frequency is $5 \mathrm{THz}$. Such sampling frequency is necessary to deal with the narrow optical pulses from the pulsed laser in Fig. 1. The optical source generates pulses at $13.6 \mathrm{MHz}$ repetition rate. These are hyperbolic secant shaped and are generated taking into account the superimposed amplitude noise, jitter of the pulses and time-width characteristics. The pulses are in accordance with the optical source employed in the experimental work in the next sections. Following the pulse laser source, DCF1 and DCF2 fibers in the analysis are characterized by the attenuation, chromatic dispersion (GVD, group velocity dispersion) and GVD slope measured by an optical network analyzer (Advantest Q7760).

The DCF model does not take into account non-linear fiber behavior as the average optical power in the laser source is kept limited to $3 \mathrm{dBm}$. The accumulated GVD in the first fiber spool is $D_{I}=-1513.1 \mathrm{ps} / \mathrm{nm}$ and relative dispersion slope is $3.29 \% / \mathrm{nm}$. The second spool of fiber, DCF2, exhibits an accumulated dispersion of $D_{2}=-3612.2 \mathrm{ps} / \mathrm{nm}$ with $3.41 \% / \mathrm{nm}$ relative dispersion slope. The resulting stretching factor is $M=1+D_{2} / D_{I}=3.38$ with negligible distortion due to chromatic dispersion slope in the $3.1-10.6 \mathrm{GHz}$ signal band. A MachZehnder interferometer is employed for electrical-to-optical conversion. The Mach-Zehnder is modeled taking into account the optical losses and the non-linearity inherent to the intrinsic half-wave $\pi$ voltage $(\mathrm{V} \pi)$. The RF input signal is amplified by $40 \mathrm{~dB}$ ( $3 \mathrm{~dB}$ noise figure) prior to the electrical-to-optical conversion. Finally, the optical signal is photodetected with a PIN photodiode taking into account thermal and shot noise, and amplified (Elect. Amp\#2) with $62 \mathrm{~dB}$ gain $(2.8 \mathrm{~dB}$ noise figure) in agreement with the amplifiers employed in the experimental work described in the next sections.

In order to evaluate the $\mathrm{Ph}$-ADC performance, a RF carrier at the first frequency of the UWB band $(f=3.146 \mathrm{GHz})$ is fed at point (2) in Fig. 1 at different power levels ranging from -39 to $-65 \mathrm{dBm}$. The resulting signals will be further compared with experimental measurements in the next section. Fig. 2 shows the resulting Ph-ADC signals at point (1) in Fig. 1 for the RF input tone at -59 and $-65 \mathrm{dBm}$ respectively. Looking at the spectrum in Fig. 2(b) and (d) it can be observed that the tone signal at $f=3.146 \mathrm{GHz}$ appears at $f / M=0.928 \mathrm{GHz}$. This confirms the time stretch with $M=3.38$.

\section{Single-ChanNel Photonic-ADC EXPERIMENTAL ANALYSIS}

Proper operation of the single-channel photonic-ADC was demonstrated in the laboratory. The experimental setup is shown in Fig. 3. A RF carrier at $f=3.146 \mathrm{GHz}$ (first frequency of the lower UWB sub-band) is employed for test at different power levels (Agilent E4438). The optical source in the setup is a Pritel femtosecond fiber laser with $13.6 \mathrm{MHz}$ repetition rate. The DCF are low loss dispersion slope compensating
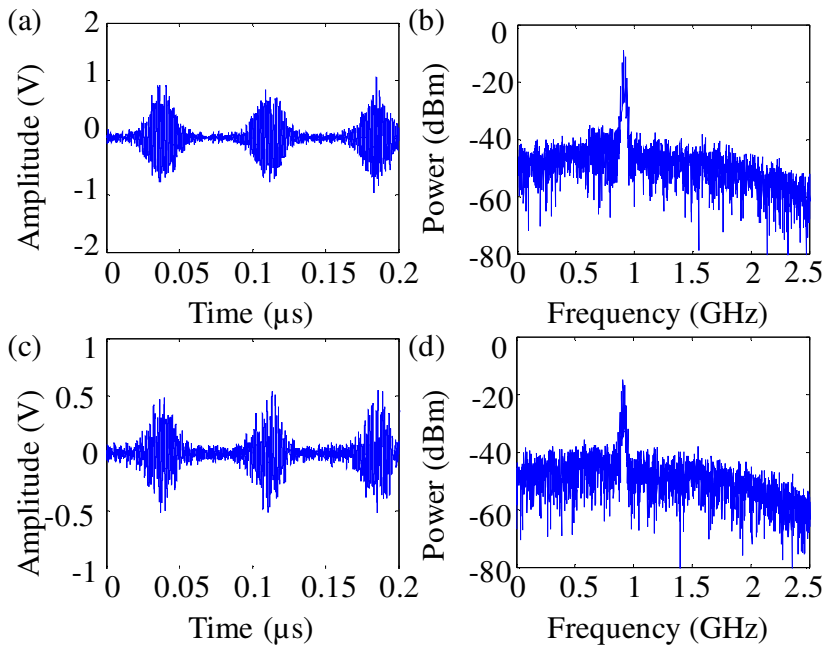

Fig. 2. Simulated time signal and electrical spectrum results for the $\mathrm{Ph}-\mathrm{ADC}$ with RF input carrier at $3.146 \mathrm{GHz}$ and: (a-b) $-59 \mathrm{dBm}$, (c-d) $-65 \mathrm{dBm}$ power respectively

modules from OFS (LLMicroDK) with accumulated dispersion $D_{l}=-1513.1 \mathrm{ps} / \mathrm{nm}$ and $D_{2}=-3612.2 \mathrm{ps} / \mathrm{nm}$. Optical amplification is introduced by low-noise erbium-doped fibre amplifier (EDFA, model Keopsys KPS-BT2-C-13-LN-FA). The saturation power is precisely set to $11 \mathrm{dBm}$ to adequately match the optical intensity to the Mach-Zehnder dynamic range limiting intermodulation and harmonics. The electro-optical modulator is a low $\mathrm{V} \pi$ modulator. The antenna amplifier (Elect. Amp\#1) has $40 \mathrm{~dB}$ gain ( $2.8 \mathrm{~dB}$ noise figure). The resulting signal is photodetected (PIN XPDV2020R, $0.7 \mathrm{~A} / \mathrm{W}$ ) and amplified with a tandem amplifier (62 dB gain). After photodetection, the signal is high-pass filtered (cut-off frequency $0.8 \mathrm{GHz}$ ) to eliminate the low frequency/high power spectrum from the optical pulse envelope. Upper cut-off frequency is $1650 \mathrm{MHz}$ which is translated to $5.61 \mathrm{GHz}$ equivalent frequency $(M=3.38)$.

Fig. 4 shows the measured electrical spectrum of the time-stretched signal at point (1) in Fig. 3. The resulting signal was sampled with a real time oscilloscope of $6 \mathrm{GHz}$ bandwidth (LeCroy SDA-760Zi) for different input power values ranging from -39 to $-65 \mathrm{dBm}$ as it is shown in Fig. 5. The amplitude levels of the experiment are in good agreement with the simulation results shown in Fig. 2. Comparing the initial frequency of the sinusoidal signal with the measured spectrum shown in Fig. 4, it is confirmed that the spectrum has been compressed by the factor $M=1+D_{2} / D_{1}=3.387$ as predicted.

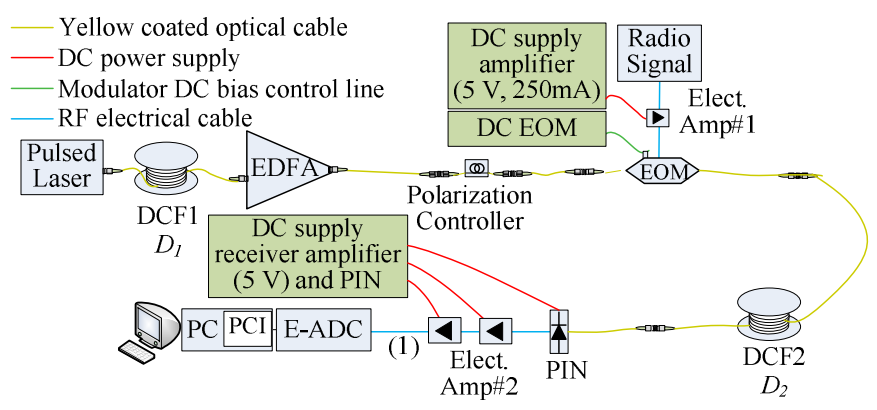

Fig. 3. Experimental single channel photonic ADC setup 
The sine peak appears at $925.48 \mathrm{MHz}$ when theoretically should be at $f_{t}=f / M=3146 \mathrm{MHz} / 3.387=928.8$, i.e. a $3.5 \mathrm{MHz}$ shift can be observed in the experiment. This is a constant shift due to the residual dispersion of the optical fibres comprising the $\mathrm{Ph}-\mathrm{ADC}$ architecture than can be corrected with calibration.

The sampled data is transferred to a PC and processed in Matlab to evaluate the signal to noise ratio (SNR). The cyclostationary period is evaluated and the carrier frequency, the phase and burst period are estimated. Afterwards, the burst envelope is estimated and the Ph-ADC SNR is computed.

Fig. 6 shows an example of the sampled signal and the results after processing. Fig. 7 shows the received signal power, the noise power and the computed SNR as a function of the burst period for the different input levels evaluated. It can be observed that the SNR degrades significantly at the edges of the pulse period. This is due to the decrease of signal power induced by the shape of the optical source pulses. The
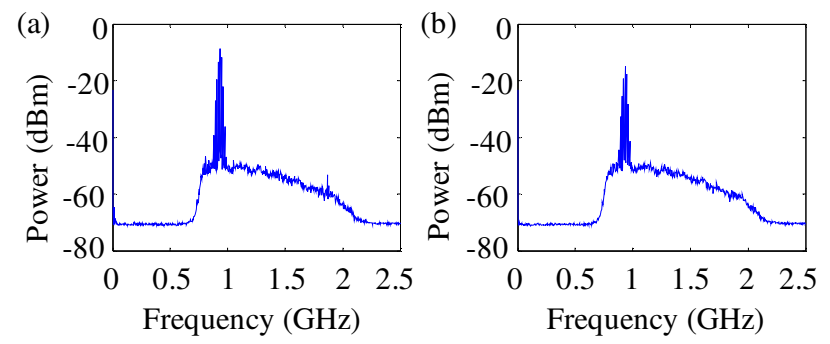

Fig. 4. Measured electrical spectrum of the Ph-ADC system with RF input carrier at $3.146 \mathrm{GHz}$ and: (a) $-59 \mathrm{dBm}$, (b) $-65 \mathrm{dBm}$ power $(\mathrm{RBW}=1 \mathrm{MHz})$
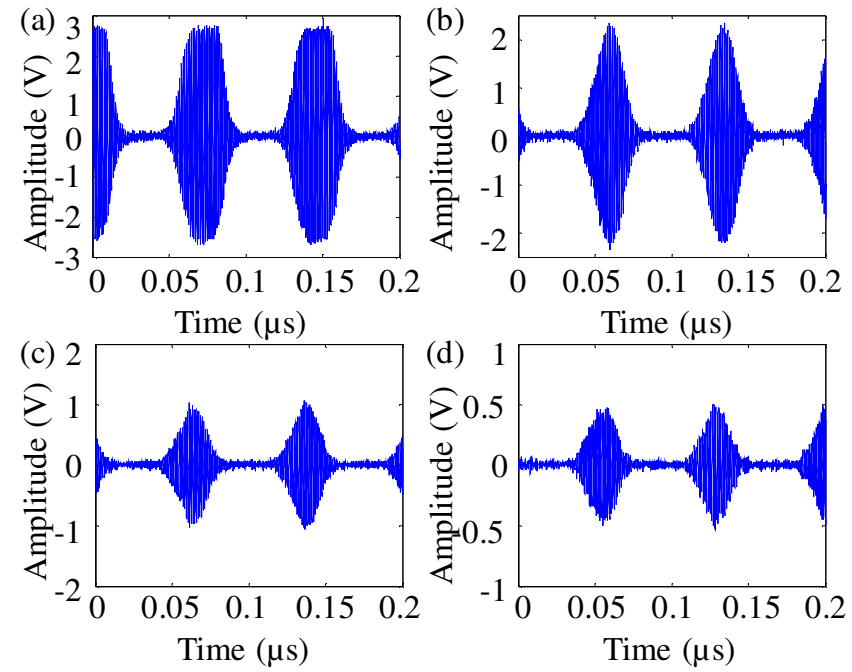

Fig. 5. Captured time signal by the $\mathrm{E}$ ADC for an RF input power of: (a) $-39 \mathrm{dBm}$, (b) $-51 \mathrm{dBm}$, (c) $-59 \mathrm{dBm}$, (d) $-65 \mathrm{dBm}$

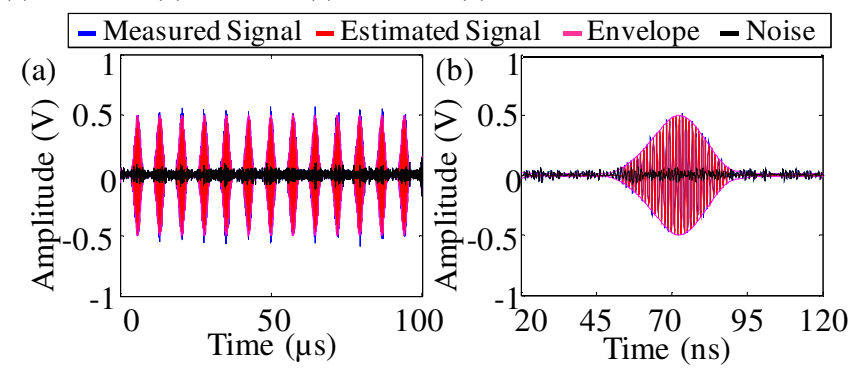

Fig. 6. (a) Processed results, envelope and estimated signal example with $\mathrm{RF}$ input power of $-65 \mathrm{dBm}$, (b) zoom
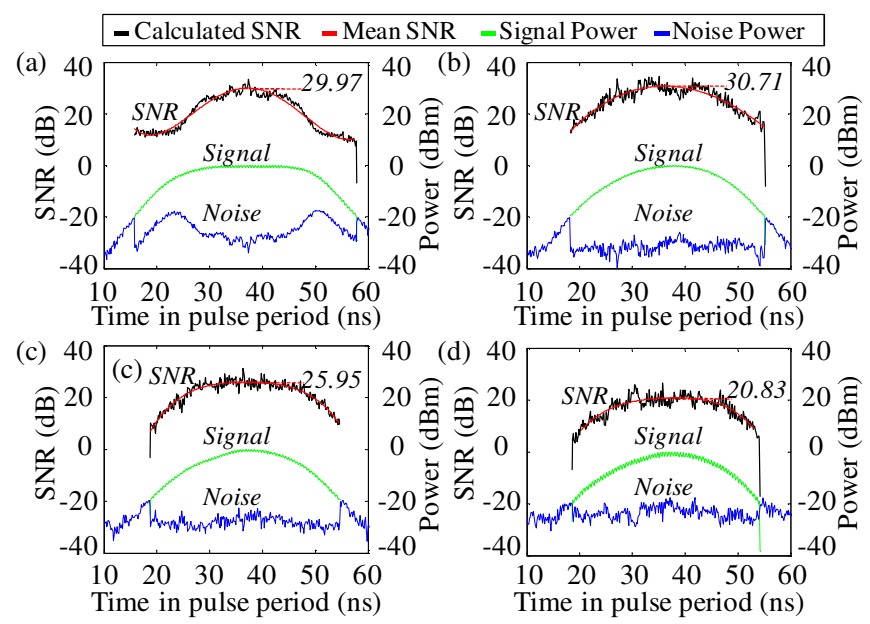

Fig. 7. SNR inside the optical pulse period for an RF input power of: (a) $-39 \mathrm{dBm}$, (b) $-51 \mathrm{dBm}$, (c) $-59 \mathrm{dBm}$, (d) $-65 \mathrm{dBm}$

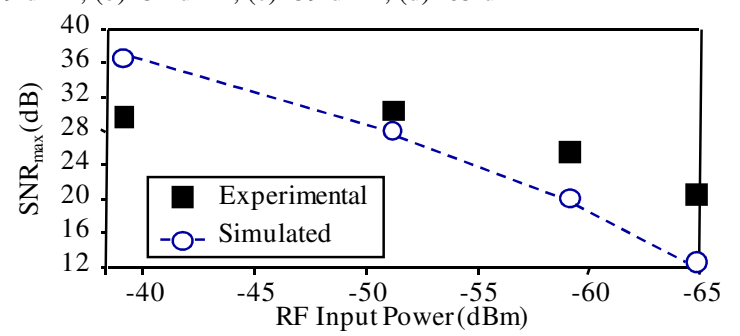

Fig. 8. Comparison of maximum SNR values obtained by simulation and experimentally vs. RF input power of the tone signal

maximum SNR obtained by simulation and experimentally are compared in Fig. 8 with good agreement.

In Fig. 8, it can be observed that for higher input power levels there is some deviation on the SNR results due to the amplitude clipping in the electrical amplifiers observed in Fig. 5(a), not considered in simulation. For lower power values, the simulated noise is bigger due to mismatches in the response of the filters used to remove the DC component coming from the optical source.

\section{Multichannel Photonic-ADC INCLUDING LOCALIZATION AND FINGERPRINTING}

The basic time-stretch $\mathrm{Ph}$-ADC principle is further developed in this section proposing a novel time- multiplexed multichannel architecture where precise control of the time alignment in the optical signal permits the simultaneous detection of a given radio signal at different spatial locations. The proposed system required synchronization of the E-ADC with the optical source and proper processing of the optical pulses coming to the E-ADC to enable the localization and fingerprinting of the detected radio signal.

The proposed photonic-ADC targets sensing the radio spectrum in a home or office environment where several sensors are located along the area to sense, and the information captured by each sensor is processed with ad-hoc fingerprinting and localization algorithms.

\section{A. Fingerprinting and localization algorithms}

Regarding the fingerprinting, the proposed algorithm is made of two sequential parts. First, the power spectral density 
is computed over the bands of interest. The integrated value over time and over each band is then put in perspective with the noise levels to give a first estimate about the presence or absence of a UWB transmission. Second, each band identified as potentially carrying an OFDM signal is then converted to baseband. The corresponding sequence should be long enough to incorporate multiple snapshots. With the selected repetition rate of the optical source, concatenating the snapshots will lead to virtual OFDM blocks exhibiting the zero-padding structure used for UWB. As proposed in [12], we then perform a power correlation on the sequence which, in case of UWB, will lead to easily identifiable trains of triangles. The period between the triangles is then estimated and compared to its theoretical value (the previously known OFDM block duration). A match will confirm the presence of a UWB signal [12].

Once the UWB transmitter is identified by the fingerprint algorithm, the proposed system estimates its position employing a specific time difference-of-arrival (TDoA) algorithm. The TDoA algorithm is a converging step, which uses the previous estimated difference of delays from each channel to compute the position. Classical differences of delays are obtained by using synchronization with the device being localized with at least two synchronized point in the infrastructure. Here is only exploited both a pure power sensing of the signal on the air and the knowledge of the used time frequency code sequence. Indeed, by matching the known hopping sequence to the sensed one, a delay is observable.
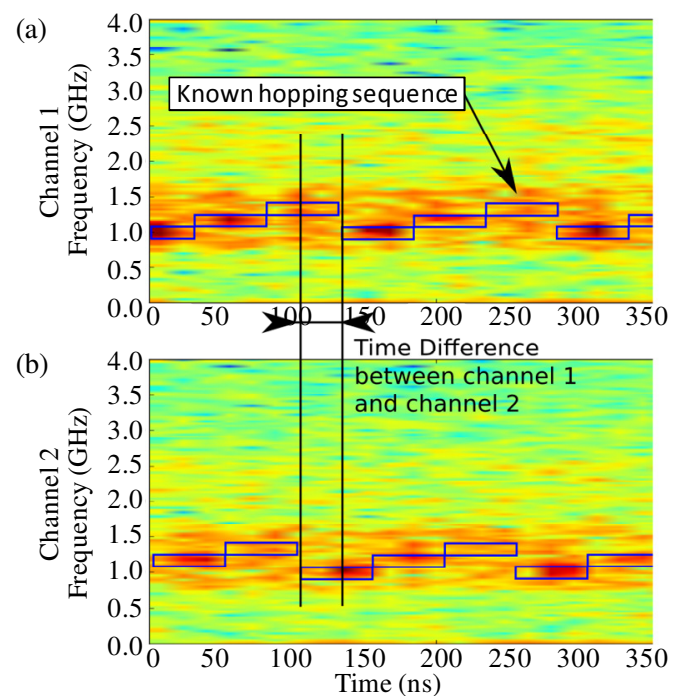

Fig. 9. Spectrogram to the received signal from two optical channels to show the delay estimation based on matching measured power and the known hopping sequence: (a) channel 1 and (b) channel 2

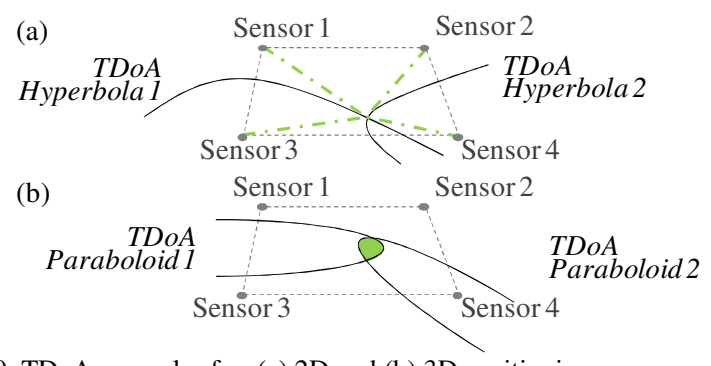

Fig. 10. TDoA examples for: (a) 2D and (b) 3D positioning
Fig. 9 shows an example of this method. By repeating on each channel, difference of delays is obtained.

The TDoA approach is enabled here by the precise timealignment in the optical domain of the signals captured in all sensors in a novel manner enabled by the time frequency power pattern sampled by the photonic-ADC. Moreover, the optical time stretching ensures a larger integration time of the sensed power increasing the delay estimation accuracy.

To exploit TDoA, least-squares based estimation schemes are the easiest and cheapest solution to perform localization from TDoAs measurements [13], so the total least square TDoA has been used [14]. Five sensors are enough to retrieve the position of the transmitters without ambiguity [8]. As shown in Fig. 10, in the case of two dimensions (2D) positioning two hyperbola can be generated with 3 sensors using the TDoA algorithm which ensures to find unique intersection. But in the case of three dimension (3D) positioning, the intersection of the two generated paraboloids returns an ambiguous set of possible solution. The inaccuracy of this area will remain ambiguous with 4 sensors. For this reason, five sensors are the minimum number required to avoid this ambiguous case. However, the position of the sensors in the given area affects the positioning accuracy that can be achieved. Consequently, before performing experimental measurements, different sensor positions have been simulated to determine the best configuration to achieve the lowest error on the TDoA. The configurations of interest are summarized in Table II.

Prior simulation analysis has been done considering the experimental demonstration room which shape is drawn in the Fig. 11 and Fig. 12 along the sensor position (white circles). The demonstration room is $4.65 \times 6.21 \mathrm{~m}$. For each sensor configuration, a simulated UWB source has been moved in and around the room (with a $0.1 \mathrm{~m}$ step), to easily detect the parabolic sector with high errors which is an intrinsic limitation of TDoA computation. For each position, the resulting difference of range has been computed 100 times using different random Gaussian error by assuming an achieved standard deviation of $0.1 \mathrm{~m}$. The root mean square (RMS) error is evaluated. In first instance, 2D positioning is evaluated for different sensors position defined with 3D coordinates $(\mathrm{x}, \mathrm{y}, \mathrm{z})$. Firstly, the TDoA algorithm is applied for 2 dimensions $(\mathrm{x}, \mathrm{y})$, in order to only take advantage of redundancy to increase the estimated position. In the first simulations (configuration A to D) the device was set at the same height at $\mathrm{z}=1.2 \mathrm{~m}$. In configuration $\mathrm{A}$, the five sensors are located at the middle width of each wall of the room at the same height $(\mathrm{z}=1.2 \mathrm{~m})$. In configuration $\mathrm{B}$, there are 4 sensors at the middle of each wall at the same height and one on the ceiling in the center of the room. In configuration $C$ the five sensors are at the corners and in configuration $\mathrm{D}$ one is on the ceiling in the middle of the room and four at the corners.

Using configuration $\mathrm{A}$, a hyperbolic undetermined area can be observed in Fig. 11(a) due to the TDoA method. Using configuration $\mathrm{B}$, the coverage area is reduced due to the 
TABLE II

SENSORS POSITION ( $\mathrm{X}, \mathrm{Y}, \mathrm{Z}$ METERS) FOR EACH CONFIGURATIONS

\begin{tabular}{c|c|c|c|c|c}
\hline \hline Conf & Sensor 1 & Sensor 2 & Sensor 3 & Sensor 4 & \multicolumn{1}{|c}{ Sensor 5 } \\
\hline A & $(3.15,0,1.2)$ & $(0,2.38,1.2)$ & $(3.15,4.75,1.2)$ & $(5.26,4,1.2)$ & $(6.21,1.2,1.2)$ \\
B & $(3.15,0,1.2)$ & $(0,2.38,1.2)$ & $(3.15,4.75,1.2)$ & $(5.26,4,1.2)$ & $(3.3,2.4,2.4)$ \\
C & $(0,0,1.2)$ & $(0,4.75,1.2)$ & $(5.26,4.75,1.2)$ & $(5.87,3.03,1.2)$ & $(6.21,0,1.2)$ \\
D & $(0,0,1.2)$ & $(0,4.75,1.2)$ & $(5.26,4.75,1.2)$ & $(3.3,2.4,1.2)$ & $(3.3,2.4,1.2)$ \\
E & $(0,0,1.8)$ & $(0,4.75,0.2)$ & $(5.26,4.75,1.8)$ & $(6.21,0,0.2)$ & $(3,3,2.4)$ \\
F & $(0,0,0.2)$ & $(0,4.75,1.8)$ & $(5.26,4.75,0.2)$ & $(6.21,0,1.8)$ & $(3,3,2.4)$ \\
\hline \hline
\end{tabular}
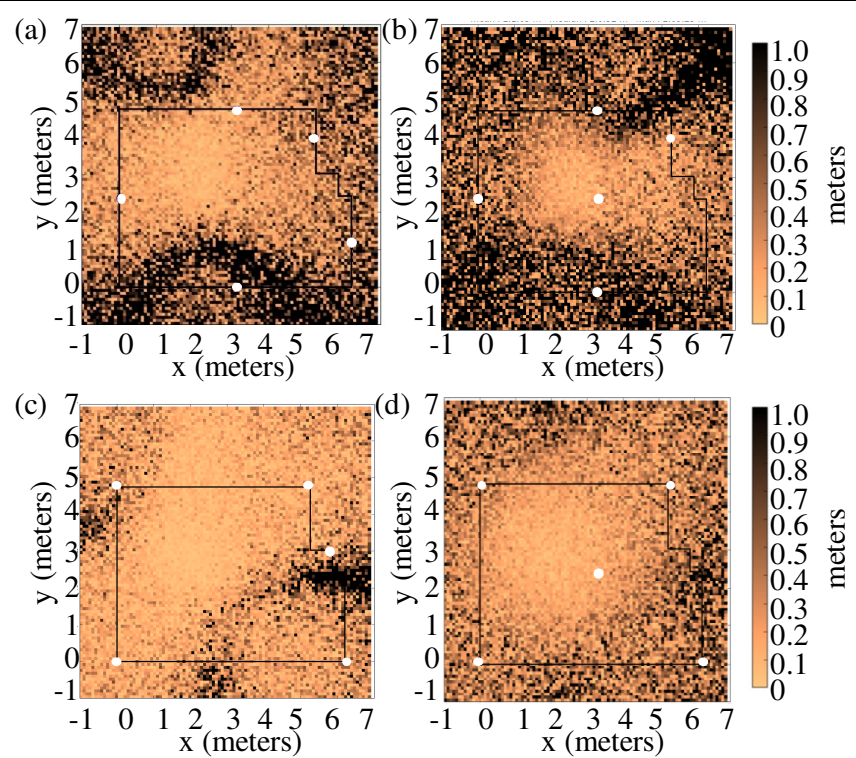

Fig. 11. Root mean square error of positioning for 5 sensors located in different configuration: (a-b) the middle of the walls, (c-d) the edges of the room

closeness of the sensors. In the corner configuration (conf. C) the positioning error is reduced, and adding a fifth sensor on the ceiling increases the coverage area (conf. D). To achieve 3D localization, it is recommended to choose different heights for each sensor. This is evaluated in configurations $\mathrm{E}$ and $\mathrm{F}$.

Locating the sensors at the same height provides a correct $(\mathrm{x}, \mathrm{y}) 2 \mathrm{D}$ positioning, but will not be accurate along z-dimension [15]. Even for 2D positioning, using different sensor heights (conf. E and F) ensures an increase in the accuracy as shown in Fig. 12. The obtained mean, median and maximum positioning errors are summarized in Table III. The performance is improved considerably by allocating the sensors at different heights. The best configuration (conf. F) achieves localization with $0.33 \mathrm{~m}$ mean and $0.23 \mathrm{~m}$ median error. Configuration $\mathrm{F}$ was selected and implemented in the demonstration experiment for comparison.

3D positioning simulations are shown in Fig. 13. These results are not as good as in Fig. 12 due to the absence of redundancy of information in the $3 \mathrm{D}$ case as well as less diversity in the repartition of sensors in the vertical plan. In the 2D case, the fifth sensor adds extra information which increases the robustness of the TDoA algorithm, but in 3D case the five sensors are useful to compute positioning. Simultaneous data captured from these five sensors in the multichannel photonic-ADC being an enabling step for the fingerprint and TDoA techniques described.
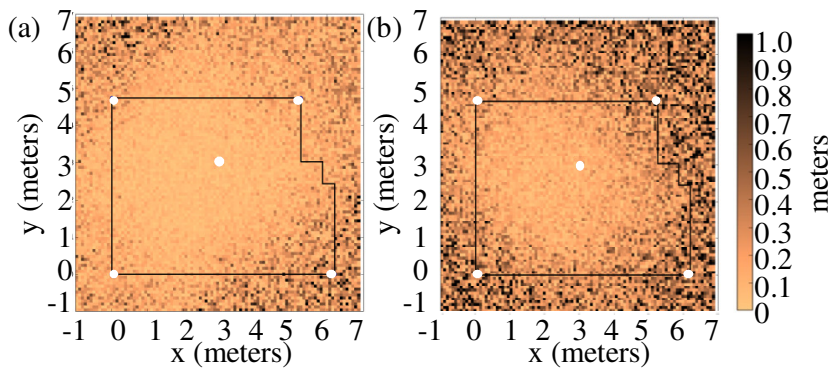

Fig. 12. Root mean square error of $2 \mathrm{D}$ positioning for 5 sensors located at different heights: (a) configuration $\mathrm{E}$, and (b) configuration $\mathrm{F}$

TABLE III

POSITIONING ABSOLUTE ERROR (CALCULATED)

\begin{tabular}{c|c|c|c}
\hline \hline Conf. & Mean Error & Median Error & Maximum Error \\
\hline A & $0.65 \mathrm{~m}$ & $0.36 \mathrm{~m}$ & $52.15 \mathrm{~m}$ \\
B & $1.05 \mathrm{~m}$ & $0.52 \mathrm{~m}$ & $69.25 \mathrm{~m}$ \\
C & $0.52 \mathrm{~m}$ & $0.31 \mathrm{~m}$ & $89.52 \mathrm{~m}$ \\
D & $0.38 \mathrm{~m}$ & $0.28 \mathrm{~m}$ & $3.48 \mathrm{~m}$ \\
E & $0.33 \mathrm{~m}$ & $0.23 \mathrm{~m}$ & $3.67 \mathrm{~m}$ \\
F & $0.33 \mathrm{~m}$ & $0.23 \mathrm{~m}$ & $2.82 \mathrm{~m}$ \\
\hline \hline
\end{tabular}
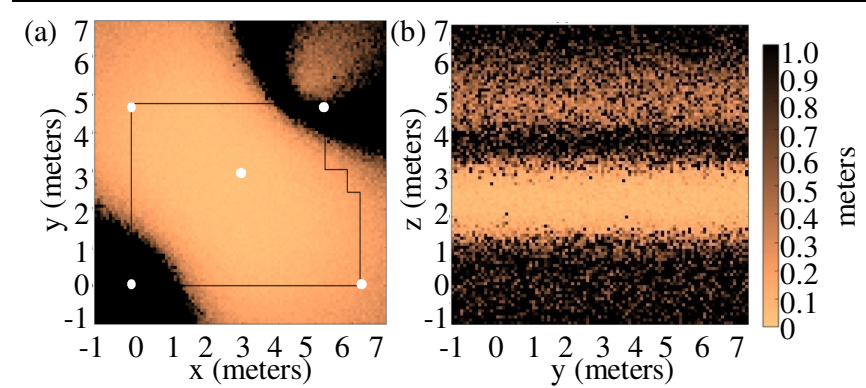

Fig. 13. Root mean square error of 3D positioning for 5 sensors located using configuration $\mathrm{F}$ : (a) on position depth $\mathrm{y}$ as a function of width $x$, and (b) on position height, $z$, as a function of depth $y$

\section{B. Experimental evaluation with multichannel Ph-ADC}

Fig. 14 shows the multichannel photonic-ADC developed. The system architecture is a multichannel extension of the single-channel Ph-ADC in Section III with full multiplexing and time-synchronization. Operation is as follows: A single optical source (Pritel femtosecond fiber laser) generates optical pulses of $270 \mathrm{~W}$ peak power, $1.6 \mathrm{ps}$ pulse width at $1 / \mathrm{e}$ and $3.233 \mathrm{MHz}$ repetition rate, which are stretched by the first spool of fibre with dispersion $D_{l}=-1513.1 \mathrm{ps} / \mathrm{nm}$ and amplified (to compensate the splitter and DCF fiber spool losses) by an EDFA (Exelite XLT-SFA-19) in 5 branches. A fibre patchcord of $10 \mathrm{~m}$ is used to feed each sensor allocated in the room according to configuration $\mathrm{F}$ as this configuration obtained the best performance for localization in the simulation studies. Each sensor comprises a UWB antenna, an RF amplifier (Elect. Amp\#1) and a Mach-Zehnder modulator. With this configuration, the optical pulses arrive to the different sensors at the same time capturing simultaneously the radio signal present in the area as a unique snapshot.

The five branches are combined together to use the same receiver elements and minimize the overall system cost. A second spool of fibre with $D_{2}=-3612.2 \mathrm{ps} / \mathrm{nm}$ is used to finish the time-stretching conversion with $M=3.38$. The stretched signal is photodetected with a PIN photodiode (XPDV2020R) 


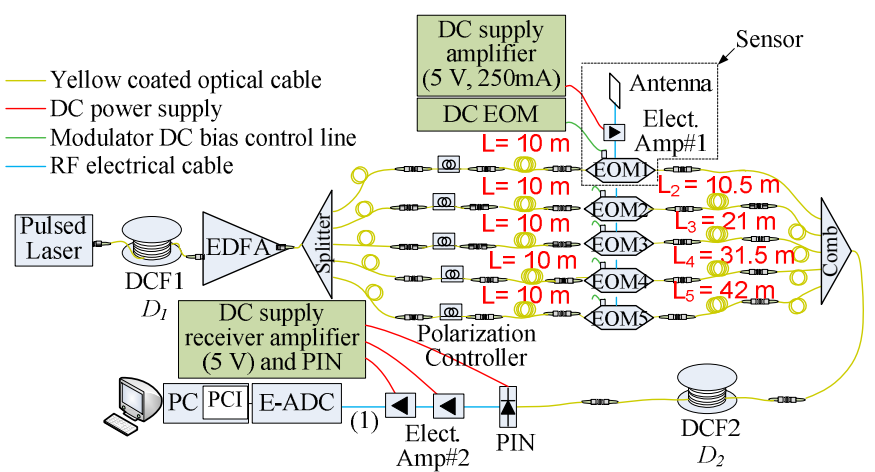

Fig. 14. Experimental setup of full-multiplexed multichannel Ph-ADC

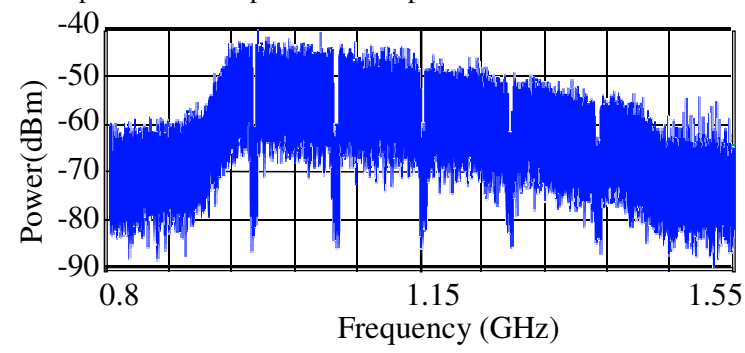

Fig. 15. UWB spectrum measured after the $\mathrm{Ph}-\mathrm{ADC}$ system $(\mathrm{RBW}=50 \mathrm{kHz})$

and amplified (Elect. Ampl\#2 with $62 \mathrm{~dB}$ gain). The signal is sampled with an E-ADC (Agilent Acqiris DC282) with a $2 \mathrm{GHz}$ front-end and a 10-bit sampling rate at $8 \mathrm{GS} / \mathrm{s}$. The UWB signal in the 3 first frequency bands (from 3.146 to $4.75 \mathrm{GHz}$ that are commercially available nowadays) after the time stretching of $M=3.38$ will be approximately $1.75 \mathrm{GHz}$, which fits with the $2 \mathrm{GHz}$ front-end specification of the E-ADC. Fig. 15 shows the measured UWB spectrum after the photonic-ADC architecture and confirms the time-stretching. Using the multiplexed architecture a single E-ADC is needed to sample all the data captured by the different sensors.

In order to avoid overlapping of the different pulses before combining them together two aspects must be taken into account. In first place, the repetition rate of the laser $\left(f_{\text {sample }}\right)$ should be calculated as:

$$
f_{\text {sample }}>\frac{1}{N \cdot T_{\text {stretched }}}
$$

where $N$ is the number of branches to combine $(N=5)$, and $T_{\text {stretched }}$ is the duration of the optical pulses once stretched with $M=3.38$. In second place, this sampling frequency has been defined such that consecutive snapshots sweep perfectly the OFDM blocks according to:

$$
f_{\text {sample }}=\frac{1}{T_{\text {OFDM }}+T_{A}}
$$

where $\mathrm{T}_{\text {OFDM }}$ is the OFDM block period and $\mathrm{T}_{\mathrm{A}}$ the aperture time of the optical pulse coming to the modulator. According to this, the repetition rate of the optical source is $3.233 \mathrm{MHz}$.

The modulated signal of each branch must be delayed before combining in order to avoid overlapping. This optical delay is achieved using different lengths of cleaved optical fiber in each branch. In the experimental setup and according to the repetition rate of the optical source, the different lengths of fibre are $L_{2}=10.5 \mathrm{~m}, L_{3}=21 \mathrm{~m}, L_{4}=31.5 \mathrm{~m}$ and $L_{5}=42 \mathrm{~m}$.

An example of the resulting results with a UWB signal

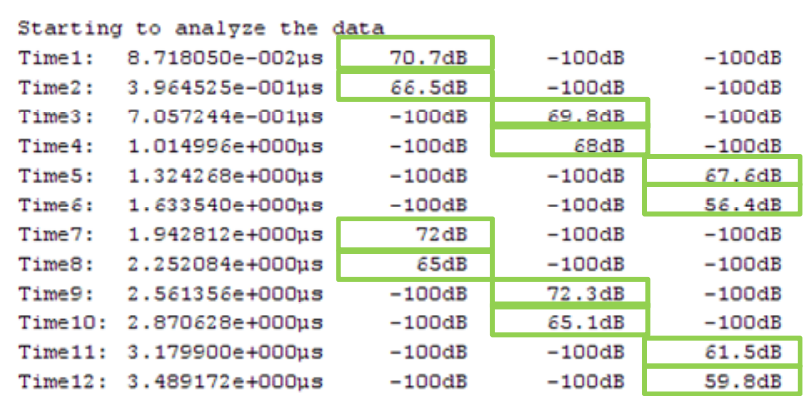

Time12: $3.489172 \mathrm{e}+000 \mu \mathrm{s} \quad-100 \mathrm{~dB} \quad-100 \mathrm{~dB} \quad 59.8 \mathrm{~dB}$

Fig. 16. Example of fingerprinting processing result of a UWB signal using time frequency code 3 ( $\mathrm{f} 1, \mathrm{f} 1, \mathrm{f} 2, \mathrm{f} 2, \mathrm{f3}, \mathrm{f3}$ )

TABLE IV

POSITION ESTIMATION IN METERS FROM A DATASET

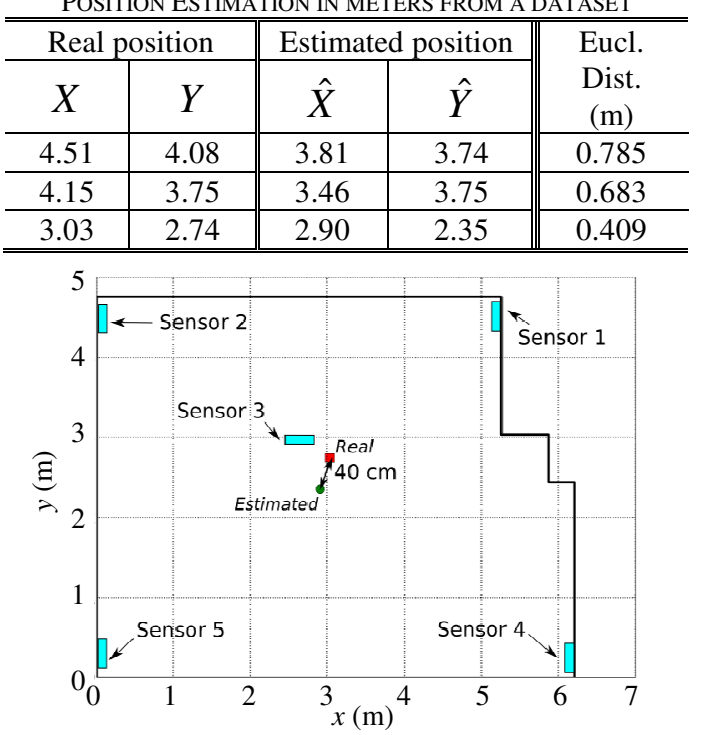

Fig. 17. Example result of positioning in the demonstration room. The red square is the real position. The green dot is the estimated

using time frequency code 3 [9] with frequency hopping in the first three channels f1, f1, f2, f2, f3, f3 is shown in Fig. 16. The $-100 \mathrm{~dB}$ labels indicate that no OFDM UWB symbols were detected in that time slot at that frequency band. If the number is different, then transmission presence was detected. In the example of Fig. 16, the frequency hopping pattern is clearly identifiable demonstrating the fingerprint of the UWB radio signal modulated in the optical pulses in the Ph-ADC.

The presence of OFDM UWB signal is a trigger to perform localization on the received data. Table IV shows some examples of the estimated position of real data from the $\mathrm{Ph}-\mathrm{ADC}$ architecture, using room configuration $\mathrm{F}$ in Table II. Three different source positions were evaluated experimentally. Fig. 17 displays one of these examples in the demonstration room with only $40 \mathrm{~cm}$ error positioning. Comparing the simulation results obtained for the same configuration $\mathrm{F}$ (Table III) where we achieved $0.33 \mathrm{~m}$ mean error, the experimental value of $0.4 \mathrm{~m}$ confirms the suitability of the algorithm.

\section{CONCLUSION}

This paper proposes and demonstrates radio sensing using a multiplexed multichannel time-stretch photonic-ADC with engineered optical and electrical amplification. Fingerprinting 
and localization algorithms were developed and tested on UWB signals detected by the Ph-ADC.

The SNR performance of a single-channel photonic-ADC is evaluated by simulation and further confirmed experimentally achieving $20.83 \mathrm{~dB}$ SNR for signals with $-65 \mathrm{dBm}$ power.

The proposed multichannel $\mathrm{Ph}-\mathrm{ADC}$ configuration enables time-spectrum gathering from different spatial points simultaneously by precise time-shift optical control, facilitating UWB transmitters' localization. Five sensors are identified as the minimum to achieve 3D localization. The full multiplexed architecture permits capturing the radio signal simultaneously at the different sensors employing a single E-ADC. The positioning error is investigated to choose the best sensor positions achieving $0.33 \mathrm{~m}$ mean error. The best positioning configuration is evaluated experimentally with a five channel multiplexed $\mathrm{Ph}$-ADC achieving $0.4 \mathrm{~m}$ error in positioning and also demonstrating fingerprinting on the optical signal identifying the frequency hopping pattern of the detected UWB signal.

\section{REFERENCES}

[1] J. Mitola III and G. Q. Maguire, "Cognitive radio: making software radios more personal,” IEEE Personal Com., Vol. 6, Issue 4, pp. 13-18, Aug. 1999.

[2] G. Dolmans, O. Rousseaux, L. Huang, T. Fu, B. Gyselinkx, S. d'Amico, A. Baschirotto, J. Ryckaert, and B.van Poucke,"UWB radio transceivers for ultra-low power and low data rate communications," IEEE International Conference on Ultra-Wideband, pp.152-157, Sept. 2007.

[3] R. Llorente, M. Morant, J. Puche, J. Romme and T. Alves, "Sensing ultra-low-power radio signals by photonic analog-to-digital conversion," 35th European Conf. on Optical Comm. ECOC09, Vienna, Sept. 2009.

[4] G. C. Valley, J. P. Hurrell, and G. A. Sefler, "Photonics analog-to-digital converters: fundamental and practical limits," SPIE European Symp. on Optics and Photonics in Security and Defence, Oct. 2004.

[5] Y. Han, B. Jalali, "Photonic time-stretched analog-to-digital converter: fundamental concepts and practical considerations," Journal of Lightwave Technology, Vol. 21. Issue 12, pp. 3085- 3103, Dec. 2003.

[6] Jd.P. Pavón, S. Shankar, V. Gaddam, K. Challapali, and C-T Chou, "The MBOA-WiMedia specification for ultra wideband distributed networks," IEEE Comm. Magazine, Vol. 44, 6, pp. 128-134, June 2006.

[7] FCC 02-48: "Revision of part 15 of the commission's rules regarding ultra-wideband transmission systems," FCC, April 2002.

[8] R. Llorente, A. Cartaxo, B. Uguen, J. Duplicy, J. Romme, J. F. Puche, D. Schmertz, Y. Lostanlen, R. Bañales and J. Marti. "Management of UWB picocell clusters: UCELLS Project approach," 2008 IEEE International Conference on Ultra-Wideband, pp. 139-142, Sept. 2008.

[9] ECMA-368: "High rate ultra wideband PHY and MAC Standard," ECMA International Standard, Dec. 2007.

[10] B. Asuri, Yan Han, B. Jalali, "Time-stretched ADC arrays," IEEE Transactions on Circuits and Systems II: Analog and Digital Signal Processing, Vol. 49, Issue 7, pp. 521-524, July 2002.

[11] J. Azaña and M. A. Muriel, "Real-time optical spectrum analysis based on the time-space duality in chirped fiber gratings", IEEE J. Quantum Electron., vol. 36, pp. 517 526, May 2000.

[12] V. Le Nir, T. van Waterschoot, M. Moonen and J. Duplicy, "Spectral monitoring and parameter estimation for ZP-OFDM signals," Proc. 17th European Signal Processing Conf. (EUSIPCO '09), Aug 2009.

[13] G. Shen and R. Zetik and R.S. Thoma, "Performance comparison of TOA and TDoA based location estimation algorithms in LOS environment," WPNC 08, 2008.

[14] M. Laaraiedh, S. Avrillon, B. Uguen, "Overcoming singularities in TDoA based location estimation using total least square" International Conf. on Signals, Circuits \& Systems, Djerba (Tunisia), Nov. 2009.

[15] K. Bronk, J. Stefanski,"Bad geometry influence on positioning accuracy in wireless network," International Conference on Computer as a Tool (EUROCON2007), Sept. 2007.

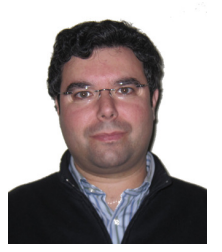

Roberto Llorente received the M.Sc. degree in Telecommunication Engineering and the Ph.D. degree from the Universidad Politécnica de Valencia (UPV), Spain, in 1998 and 2006, respectively. Since then, he has been in research positions within the university, and in 2002 he joined the Valencia Nanophotonics Technology Center (VNTC).

Currently, he is an Associate Professor of the UPV, teaching radio-communications-related subjects and he is the head of the optical access group at VNTC. He has been the Technical Responsible of the European project FP6-IST-UROOF, and Coordinator of FP7-IST-UCELLS and FP7-ICT-FIVER projects, from January 2008 and 2010 respectively. His research interest includes optical and electro-optical processing techniques in areas of transmission systems and hybrid wireless-optical access networks.

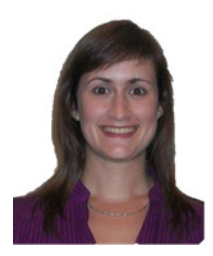

Maria Morant received the M.Sc. degree in telecommunication engineering from the Universidad Politécnica de Valencia, Spain, in 2008. She is currently working towards the Ph.D. degree in telecommunications in the optical access group at the Valencia Nanophotonics Technology Center.

Since 2006, she has been collaborating with the VNTC in UWB radio signals propagation over optical fiber and worked on the European projects FP6-IST-UROOF, FP7-ISTUCELLS and FP7-ICT-FIVER. Her current research areas of interest include UWB communications on optical fiber networks.

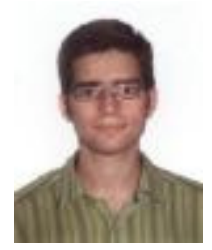

José J. Puche received the degree in telecommunication engineering from the Universidad Politécnica de Valencia, Spain, in 2007. He is currently working in DAS Photonics, Valencia, Spain.

Since 2008 he is working on European project FP7ICT-UCELLS in photonic analog to digital converters for sensing ultra-wide band signals.

Jac Romme received the M.Sc. degree in electrical engineering from Eindhoven University of Technology in 2000 and the Ph.D. degree from Graz University of Technology in 2008. Since 2000, he has been working as a research engineer at IMST GmbH, Kamp-Lintfort, Germany.

He has been involved in various UWB projects on localization and data communication. His research interests are statistical signal processing, nonlinear detectors, channel equalization, and forward error control.

Nicolas Amiot received his Dipl.-Ing. degree in Electronics and Signal Processing from the National Institute of Applied Sciences (INSA) of Rennes, France in 2007. His current research areas are localization in wireless network and UWB communications.

Since 2008 he is involved in European project FP7-ICT-UCELLS in localization of UWB devices.

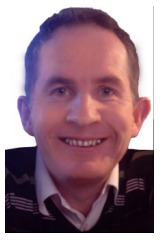

Bernard Uguen received his Dipl.-Ing. and his Ph.D degree in Electronics and Signal Processing from the National Institute of Applied Sciences (INSA) of Rennes, France, in 1988 and 1992 respectively. From 1993 to 2006 he has been an Associate Professor at the Electronics and Communications Systems (ESC) Department of the INSA of Rennes.

In Sept. 2006 he joins University of Rennes 1 where he is currently professor. He belongs, as a researcher, to the Rennes institute for Electronics and Telecommunications. His main research interests are electromagnetic and diffraction theories, propagation modeling, radar and UWB technology. He has been involved in the application of 3D ray tracing for analysis of UWB localization systems in multipath channel. His current interests are related to wireless UWB communications and localization.

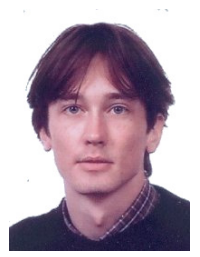

Jonathan Duplicy received Electrical Engineering degree from the Université Catholique de Louvain (UCL), Belgium, in 2003. Between 2003 and 2008, he was with the Communications and Remote Sensing Laboratory of UCL working on multi-user multi-antenna systems. In 2006 he completed a master degree in management at the Solvay Business School, Brussels. He is currently with Agilent Technologies Measurement Research Laboratory. His research interests focus on signal processing for digital communications with focus on advanced wireless technologies. 